Memorias del VII Encuentro Nacional de Experiencias en la Enseñanza de la Biología y la Educación Ambiental y II Congreso Nacional de Investigación en la Enseñanza de la Biología

\title{
LA REPRESENTACIÓN CIENTÍFICA ESCOLAR DENTRO DEL DISCURSO DE LOS Y LAS JÓVENES WAYUU SOBRE LAS PLANTAS.
}

\section{THE SCIENTIFIC SCHOOL REPRESENTATION INSIDE THE SPEECH OF THE YOUNG PERSONS WAYUU ON THE PLANTS.}

\author{
Gina Aldana ${ }^{1}$ \\ Andrés Venegas ${ }^{2}$ \\ Diana Bautista ${ }^{3}$
}

\section{Resumen}

El presente artículo aborda la representación científica escolar que emergió del trabajo de investigación "Representaciones Sociales de las y los Jóvenes Wayúu en torno a sus Plantas: El Saber de Nuestras Plantas Wayúu". De tal forma, el articulo atiende a explicaciones de orden escolar que se manifiestan en el análisis e interpretación por medio de la triangulación de tres instrumentos: 1) Taller de implementación acerca de las plantas y reconocimiento de plantas locales, denominado, ¿Qué tanto sabes de las plantas de tú alrededor?; 2) Caracterización de los pobladores; 3) Entrevistas semi-estructuradas. Asimismo se realizó el relevamiento bibliográfico con referencia a las plantas Wayúu que permitió generar un contexto histórico para los saberes ancestrales y una visión más específica de la cultura Wayúu.

Palabras Clave: Plantas Wayúu, Saber Escolar, Cosmovisión, Representaciones Sociales.

\section{Abstract}

The present article approaches the scientific school representation that emerged of the work of investigation " Social Representations of them and the Young persons Wayúu concerning his Plants: To know of Our Plants Wayúu ". Of such a form, the article attends to explanations of school order that demonstrate in the analysis and interpretation by means of the triangulation of three instruments: 1) Workshop of implementation brings over of the plants and recognition, called of local plants, what so much do you know of the plants of you around?; 2) Characterization of the settlers; 3) semi-structured Interviews. Likewise the bibliographical report was realized by reference to the plants Wayúu that a historical context allowed to

\footnotetext{
${ }^{1}$ Licenciada en Biología. Universidad Distrital. Docente del resguardo Indígena Perratpu.

${ }^{2}$ Doctorado Interinstitucional en Educación. cDr en Educación. Docente Universidad Distrital.

${ }^{3}$ Licenciada en Biología. Universidad Distrital francisco josé de caldas-. Centro de investigaciones y desarrollo científico.
} 
Memorias del VII Encuentro Nacional de Experiencias en la Enseñanza de la Biología y la Educación Ambiental y II Congreso Nacional de Investigación en la Enseñanza de la Biología

generate for the ancient knowledge and the most specific vision of the culture Wayúu.

Keywords: Plants Wayuu, Saber School, Worldview, Social Representations.

\section{Introducción}

Jodelet y Guerrero (2000) explican como en las representaciones sociales (RS) son fundamentales en la experiencia cotidiana, asimismo que ellas manifiestan una imagen temporal, específica y única de la sociedad, donde toman un papel importante los intercambios de lenguaje, los procesos de comunicación y la cultura.

En este sentido, el estudio sobre las plantas Wayúu con estudiantes de grado séptimo permitió adjudicar un papel a la enseñanza de las ciencias naturales en el contexto indígena. Para ello se adelantó un proceso de triangulación que permitió la caracterización y decantación de los discursos de los estudiantes Wayúu en cuanto a las plantas bajo la rejilla de las representaciones sociales.

Ahora bien, al interior del estudio de las representaciones sociales aparece la teoría del núcleo central (Abric, 2001), la cual fue asumida en la investigación. De esta manera, el núcleo central de las representaciones sociales en torno al saber sobre las plantas Wayúu del grupo de estudio fue la RS denominada cosmovisión, la cual se constituye a partir de una serie de saberes, conocimientos y elementos sociales que la configuran como representación central (Véase figura 1) y dan cuenta de la forma de ver el mundo, por medio de las plantas.

Esta representación es autónoma, pues a partir de ella se organizan las otras representaciones, económica, alimenticia, medicinal, como también la científica escolar, la cual hace referencia a explicaciones en torno a las plantas teniendo como base ciertos aspectos que se desarrollan en la clase de ciencias naturales dentro del contexto occidental.




Bio-grafia Escritos sobre La Biologia y su Enseñanza.

Edición Extra-Ordinaria. ISSN 2027-1034 P.p 607 - 613

Memorias del VII Encuentro Nacional de Experiencias en la Enseñanza de la Biología y la Educación Ambiental y II Congreso Nacional de Investigación en la Enseñanza de la Biología

Ilustración 1. Representación social de los y las jóvenes Wayúu sobre sus plantas locales.

\section{Metodología y grupo de estudio.}

La metodología empleada para obtener los resultados aquí expuestos fue de corte cualitativo con un enfoque etnográfico. La investigación se desarrolló con once estudiantes de origen cultural Wayúu, 2 hombres y 9 mujeres, asistentes a la institución educativa Luis Robles ubicado en el municipio de Camarones, la Guajira. Todos ellos se encontraban cursando el grado séptimo para el 2011, año en el cual se desarrolló la fase de campo. A continuación se describen los instrumentos:

El Instrumento I: ¿Qué tanto sabes de las plantas de tú alrededor?, fue diseñado con el objetivo de determinar las representaciones sociales sobre las plantas que poseen los estudiantes, se dividió en dos partes, la primera es un cuestionario escrito donde los jóvenes responden preguntas acerca de lo que saben sobre sus plantas locales. En este instrumento se realizan preguntas acerca de cómo conciben el origen de las plantas, qué tipo de plantas existen y cuál es el uso que le dan los Wayúu a las plantas. La segunda parte está diseñada para que los estudiantes plasmen las plantas a través de dibujos.

El Instrumento II: Taller implementación acerca de las plantas y reconocimiento de plantas locales, se diseñó para reconocer las representaciones de los estudiantes desde un ambiente más informal sin dejar de estar presente el contexto escolar. Este consta de tres partes: una lectura sobre como el hombre clasifica las plantas, en este sentido se pretende mostrar dos perspectivas sobre su clasificación, una visión científica occidental y otra Wayúu; la segunda parte consistió en una salida de campo a las comunidades cercanas; en la tercera parte los estudiantes plasmaron lo discutido en el recorrido por medio de la construcción de un herbario que debía contener las plantas con información referente al nombre en español y en Wayunnaiki, las historias que se discutieron, los usos, etc.

El instrumento III: Caracterización de los pobladores, es una entrevista semiestructurada diseñada con el objetivo de observar los rasgos principales del estilo de vida que llevan los integrantes de la comunidad.

\section{Resultados: Representación Científica Escolar y el Discurso de los y las Jóvenes.}

Los discursos de los estudiantes sobre las plantas exponen una representación científico escolar enmarcada en conceptos y afirmaciones propias de la clase de ciencias naturales, como de los conocimientos científicos occidentales. Es decir, algunas de las representaciones que tienen los estudiantes han sido forjadas a 
Memorias del VII Encuentro Nacional de Experiencias en la Enseñanza de la Biología y la Educación Ambiental y II Congreso Nacional de Investigación en la Enseñanza de la Biología

través del tiempo mediante el aprendizaje en la escuela occidental, pues cabe mencionar que la institución Luis $A$ Robles no es un colegio indígena, lo cual hace que el currículo este orientado a lo establecido por las directrices estándar del Ministerio de educación para Colombia.

Esta representación emerge en el instrumento I y III en algunas respuestas asociadas a la pregunta, ¿Sabes qué es una planta? Éstas mostraron una relación con el conocimiento escolar, ocupando un porcentaje del 28\% (Véase llustración 2). Tomando de esta forma el valor más alto dentro de las representaciones de los jóvenes. En correlación con lo anterior, los estudiantes expresan que las plantas son: "un ser vivo", "las plantas son seres que se producen viven y mueren", "son las que se reproducen viven y mueren". De este modo, se infiere que los jóvenes Wayúu exhiben conceptos de las ciencias escolares al referirse a las plantas. Reflejando la asimilación de conceptos occidentales al margen de una negociación de saberes con los conocimientos propios de la comunidad indígena.

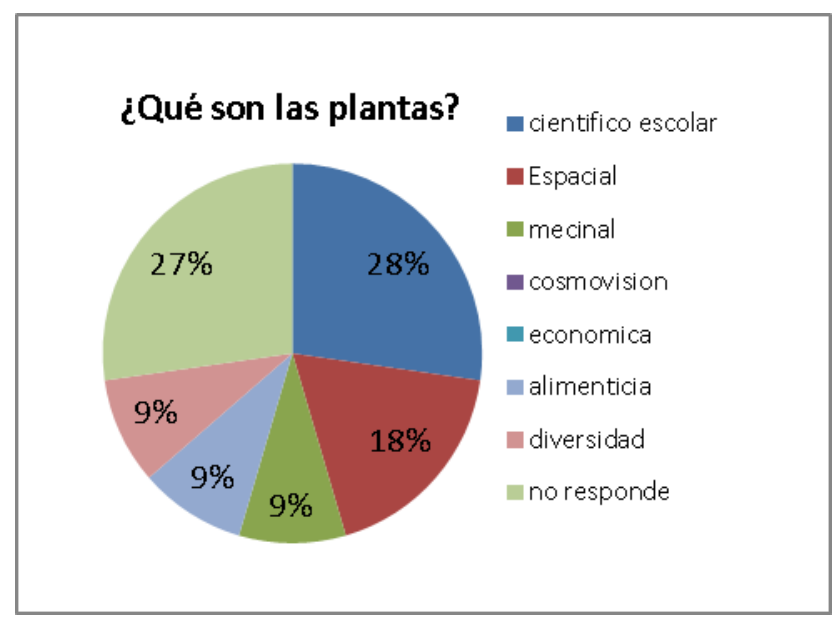

Ilustración 2. Porcentaje de respuestas referidas a las representaciones.

El aspecto vislumbrado anteriormente revela la incidencia que poseen la clase de ciencias y los libros textos escolares en los procesos de asimilación cultural encaminados por la escuela. Un ejemplo claro es la definición que manejan los estudiantes en torno a los seres vivos pues ésta se encuentra en concordancia con lo expuesto por los libros de texto,

Los seres vivos son los que comparten algunas características o cualidades. Están conformados por células, poseen movimiento, se reproducen... (Salamanca, 2003. p. 23)

Los seres vivos son los que tienen la Facultad de crecer, todo ser vivo está sujeto, una vez que nace, a un proceso de crecimiento que varía en 
Memorias del VII Encuentro Nacional de Experiencias en la Enseñanza de la Biología y la Educación Ambiental y II Congreso Nacional de Investigación en la Enseñanza de la Biología

cantidad y rapidez. Facultad de reproducirse. También la vida declina lentamente aun en el caso que no intervenga ninguna causa circunstancial, ningún accidente. Este proceso de la vida tiene un fin inevitable. La muerte... (Zweig, 1962. p.60)

De forma semejante, las respuestas a la pregunta ¿De dónde vienen las plantas? del instrumento I atañen a un contexto científico escolar, resultado que se respalda con un porcentaje del 43\% (Véase Ilustración 3), encontrando expresiones tales como, "vienen de las semillas". Esta definición entra en concordancia con lo expuesto por Salamanca (2003) donde a partir de las semillas se forma parte del fruto y al germinar en condiciones adecuadas dan lugar a las plantas.

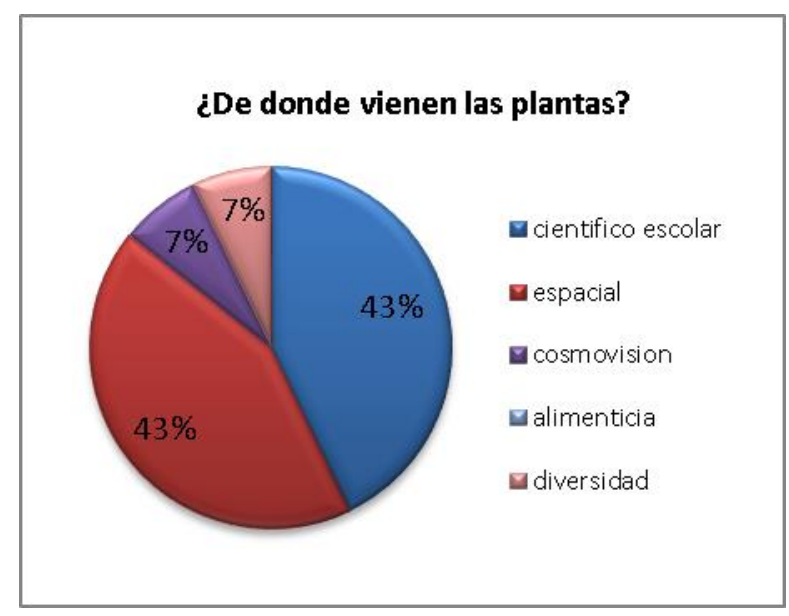

Ilustración 3. Porcentaje de respuestas referido a las representaciones exhibidas a través de la pregunta ¿De dónde vienen las plantas?

También se observó la representación científico escolar a la hora de hablar sobre las partes que poseen las plantas, aspecto retomado en las clases de ciencias, pues se evidencia que un $26 \%$ de los estudiantes identifican la raíz como parte de ellas, seguido por el tallo con un $23 \%$, hojas $19 \%$ y flor $12 \%$. En menor porcentaje se reconoce el fruto como parte de las plantas con un $9 \%$, el tronco, con un $4 \%$, y las ramas con un $3 \%$.

Lo anterior revela que las representaciones de los y las jóvenes Wayúu están orientadas por estos conocimientos pues la clasificación expuesta es enseñada a lo largo del proceso escolar en la Institución Luis A Robles, partiendo de los contenidos de los libros de texto donde se trata la morfología de las plantas, como lo exhibe Salamanca (2003).

Las raíces son las que anclan la planta y absorben el agua y nutrientes de la tierra, el tallo que es la parte de la planta que crece en sentido contrario 
Memorias del VII Encuentro Nacional de Experiencias en la Enseñanza de la Biología y la Educación Ambiental y II Congreso Nacional de Investigación en la Enseñanza de la Biología

de la raíz, es decir, hacia arriba. Del tallo salen las ramas, las hojas, las flores y frutos. Las hojas son la parte de la planta que realiza el proceso de fotosíntesis. Las flores son las que funcionan como órganos reproductores cuyo resultado se refleja en el fruto. La semilla es una estructura protectora que contiene un embrión y un suministro de alimento. (p.37)

\section{Conclusiones}

Se estudiaron las representaciones sociales, puesto que ellas, funcionan como un sistema de interpretación de la realidad, el cual rige las relaciones de los individuos en sus contextos, además determinan sus comportamientos o prácticas, de esta forma, los estudiantes exhibieron conocimientos de sus contextos, en especial sobre el uso de las plantas como medicina y su cosmovisión. Así las representaciones conciernen a los conocimientos del sentido común junto a los procesos cognitivos y culturales (Jodelet ,2000).

En este sentido, el trabajo sirvió para vislumbrar las representaciones que tienen los y las jóvenes hacia las plantas que encuentran en su entorno, obteniendo resultados que se analizaron mediante la teoría del núcleo central propuesta por Abric (2001), la cual evidenció como núcleo central la representación cosmovisión, pues esta es la forma de ver el mundo, siendo una representación generadora de otras.

De este modo, la cosmovisión abarca varios aspectos para los y las jóvenes Wayúu, pues constituye un sistema de creencias propias, donde las plantas son pilares en la forma de concebir el mundo, puesto que son seres que dotaron de vida a los Wayúu.

Asimismo, para los estudiantes las plantas poseen poderes espirituales que curan o enferman según la situación, encontrando que existen plantas buenas y malas, pues fueron Wayúu.

Además las plantas poseen historias ancestrales, las cuales refieren los abuelos 0 personas mayores para la enseñanza de normas y conductas morales, como se presenta las narraciones sobre el Wanuluu y el jinete que rondaba en las noches. (Gonzales, 2006)

Alrededor de la cosmovisión giran otras representaciones sociales más flexibles, lo cual permite que algunas de ellas entren en contradicción. La representación científico escolar exhibida por los jóvenes Wayúu, mostró explicaciones en torno a las plantas basadas en múltiples aspectos aprendidos en la clase de ciencias naturales, donde estas son concebidas como seres vivos, provenientes de semillas, una postura de occidente. 
Memorias del VII Encuentro Nacional de Experiencias en la Enseñanza de la Biología y la Educación Ambiental y II Congreso Nacional de Investigación en la Enseñanza de la Biología

De este modo, los estudiantes refieren que las plantas nacen, crecen se reproducen y mueren. Argumento forjado a través del tiempo mediante la enseñanza de las ciencias naturales y el uso de las definiciones que ostentan los libros de texto.

En concordancia a lo anterior, se vislumbra que los y las jóvenes Wayúu revelan como las clases de ciencias tienen importancia en sus explicaciones del mundo natural en especial con referencia a las plantas. No obstante, es preocupante el hecho que los procesos de asimilación que se dan en la clase de ciencias estén impactando de tal forma los saberes tradicionales y ancestrales de la comunidad Wayúu, pues estos no entran en negociación en este espacio académico al ser invisibilizados, aspecto que empieza a mostrarse a través de las representaciones.

\section{Bibliografía}

Jodelet D., \& Guerrero A. (2000). Develando la cultura. Estudios en representaciones sociales. Universidad nacional autónoma de México.

Abric, J.C (2001). Prácticas sociales y representaciones. Ediciones Coyacan, s.a de c.v. México. Primera edición

Salamanca. G., \& Magda. N., (2003) Inteligencia científica 6 libro de texto de ciencia naturales. Colombia. Editorial voluntad.

Zweig. E., (1962) Enciclopedia básica de consulta fácil. Buenos aires. Editores enciclopedia británica. Inc.

Gonzales. R. O. (2006) Uso y tratamiento del wayunaiki en dos instituciones etnoeducativas wayuu. Universidad mayor de san simón programa de educación intercultural bilingüe para los países andinos. Cochabamba. Bolivia. 